\title{
Simulation of the performance and scalability of message passing interface (MPI) communications of atmospheric models running on exascale supercomputers
}

\author{
Yongjun Zheng and Philippe Marguinaud \\ Centre National de Recherches Météorologiques, Météo France, Toulouse, France \\ Correspondence: Yongjun Zheng (yongjun.zheng@meteo.fr) \\ Received: 28 November 2017 - Discussion started: 22 January 2018 \\ Revised: 2 July 2018 - Accepted: 17 July 2018 - Published: 22 August 2018
}

\begin{abstract}
In this study, we identify the key message passing interface (MPI) operations required in atmospheric modelling; then, we use a skeleton program and a simulation framework (based on SST/macro simulation package) to simulate these MPI operations (transposition, halo exchange, and allreduce), with the perspective of future exascale machines in mind. The experimental results show that the choice of the collective algorithm has a great impact on the performance of communications; in particular, we find that the generalized ring- $k$ algorithm for the alltoallv operation and the generalized recursive- $k$ algorithm for the allreduce operation perform the best. In addition, we observe that the impacts of interconnect topologies and routing algorithms on the performance and scalability of transpositions, halo exchange, and allreduce operations are significant. However, the routing algorithm has a negligible impact on the performance of allreduce operations because of its small message size. It is impossible to infinitely grow bandwidth and reduce latency due to hardware limitations. Thus, congestion may occur and limit the continuous improvement of the performance of communications. The experiments show that the performance of communications can be improved when congestion is mitigated by a proper configuration of the topology and routing algorithm, which uniformly distribute the congestion over the interconnect network to avoid the hotspots and bottlenecks caused by congestion. It is generally believed that the transpositions seriously limit the scalability of the spectral models. The experiments show that the communication time of the transposition is larger than those of the wide halo exchange for the semi-Lagrangian method and the allreduce in the generalized conjugate residual (GCR) iterative solver for the semi-implicit method below $2 \times 10^{5} \mathrm{MPI}$
\end{abstract}

processes. The transposition whose communication time decreases quickly with increasing number of MPI processes demonstrates strong scalability in the case of very large grids and moderate latencies. The halo exchange whose communication time decreases more slowly than that of transposition with increasing number of MPI processes reveals its weak scalability. In contrast, the allreduce whose communication time increases with increasing number of MPI processes does not scale well. From this point of view, the scalability of spectral models could still be acceptable. Therefore it seems to be premature to conclude that the scalability of the grid-point models is better than that of spectral models at the exascale, unless innovative methods are exploited to mitigate the problem of the scalability presented in the grid-point models.

\section{Introduction}

Current high-performance computing (HPC) systems have thousands of nodes and millions of cores. According to the 49th TOP500 list (https://www.top500.org, last access: 16 August 2018) published on 20 June 2017, the fastest machine (Sunway TaihuLight) had over 10 million cores with a peak performance of approximately 125 PFlops ( 1 PFlops = $10^{15}$ floating-point operations per second), and the second HPC (Tianhe-2) is made up of 16000 nodes and has more than 3 million cores with a peak performance of approximately 55 PFlops. It is estimated that in the near future, HPC systems will dramatically scale up in size. Next decade, it is envisaged that exascale HPC system with millions of nodes and thousands of cores per node, whose peak perfor- 
mance approaches to or is beyond 1 EFlops $\left(1\right.$ EFlops $=10^{3}$ PFlops), will become available (Engelmann, 2014; Lagadapati et al., 2016). Exascale HPC poses several challenges in terms of power consumption, performance, scalability, programmability, and resilience. The interconnect network of exascale HPC system becomes larger and more complex, and its performance which largely determines the overall performance of the HPC system is crucial to the performance of distributed applications. Designing energy-efficient costscalable interconnect networks and communication-efficient scalable distributed applications is an important component of HPC hardware/software co-design to address these challenges. Thus, evaluating and predicting the communication behaviour of distributed applications is obligatory; it is only feasible by modelling the communications and the underlying interconnect network, especially for the future supercomputer.

Investigating the performance of distributed applications in future architectures and the impact of different architectures on the performance by simulation is a hardware/software co-design approach to pave the way to exascale HPCs. Analytical interconnect network simulation based on an analytical conceptual model is fast and scalable, but it comes at the cost of accuracy owing to its unrealistic simplification (Hoefler et al., 2010). Discrete event simulation (DES) is often used to simulate the interconnect network, and it provides high fidelity since the communication is simulated at a more detailed level (e.g., flit, packet, or flow levels) to take into account congestion (Janssen et al., 2010; Böhm and Engelmann, 2011; Dechev and Ahn, 2013; Acun et al., 2015; Jain et al., 2016; Wolfe et al., 2016; Degomme et al., 2017; Mubarak et al., 2017). Sequential DES lacks scalability owing to its large memory footprints and long execution time (Degomme et al., 2017). Parallel DES (PDES) is scalable since it can reduce the memory required per node, but its parallel efficiency is not very good because of frequent global synchronization of conservative PDES (Janssen et al., 2010) or high rollback overhead of optimistic PDES (Acun et al., 2015; Jain et al., 2016; Wolfe et al., 2016). Generally, the simulation of distributed applications can be divided into two complementary categories: offline and online simulations. Offline simulation replays the communication traces from the application running on a current HPC system. It is sufficient to understand the performance and discover the bottleneck of fully distributed applications on the available HPC system (Tikir et al., 2009; Noeth et al., 2009; Núñez et al., 2010; Dechev and Ahn, 2013; Casanova et al., 2015; Acun et al., 2015; Jain et al., 2016; Lagadapati et al., 2016); however, is not very scalable because of the huge traces for numerous processes and limited extrapolation to future architecture (Hoefler et al., 2010; Núñez et al., 2010). Online simulation has full scalability to future systems by running the skeleton program on top of simulators (Zheng et al., 2004; Janssen et al., 2010; Engelmann, 2014; Degomme et al., 2017), but it has the challenge of developing a skeleton program from a complex distributed application. Most simulations in the aforementioned literature have demonstrated the scalability of simulators. The simulator xSim (Engelmann, 2014) simulated a very simple message passing interface (MPI) program, which only calls MPI_Init and MPI_Finalize without any communication and computation, up to $2^{27}$ processes. For collective MPI operations, Hoefler et al. (2010) obtained an MPI_Allreduce simulation of 8 million processes without consideration of congestion using LogGOPSim, Engelmann (2014) achieved an MPI_Reduce simulation of $2^{24}$ processes, and Degomme et al. (2017) demonstrated an MPI_Allreduce simulation of 65536 processes using SimGrid. For simulations at application level, Jain et al. (2016) used the TraceR simulator based on CODES and ROSS to replay $4.6 \times 10^{4}$ process traces of several communication patterns that are used in a wide range of applications. In addition, Mubarak et al. (2017) presented a $1.1 \times 10^{5}$ process simulation of two multigrid applications. However, to the best of our knowledge, there is no exascale simulation of complex communication patterns such as the MPI transposition (Multiple simultaneous MPI_Alltoallv) for the spectral method and the wide halo exchange (the width of a halo may be greater than the subdomain size of its direct neighbours) for the semi-Lagrangian method used in atmospheric models.

With the rapid development of increasingly powerful supercomputers in recent years, numerical weather prediction (NWP) models have increasingly sophisticated physical and dynamical processes, and their resolution is getting higher and higher. Nowadays, the horizontal resolution of a global NWP model is in the order of $10 \mathrm{~km}$. Many operational global spectral NWP models such as IFS at ECMWF, ARPEGE at Météo France, and GFS at NCEP are based on the spherical-harmonics transform method that includes Fourier transforms in the zonal direction and Legendre transforms in the meridional direction (Ehrendorfer, 2012). Moreover, some regional spectral models such as AROME at Météo France (Seity et al., 2011) and RSM at NCEP (Juang et al., 1997) use the bi-Fourier transform method. The Fourier transforms can be computed efficiently by fast Fourier transform (FFT) (Temperton, 1983). Even with the introduction of fast Legendre transform (FLT) to reduce the growing computational cost of increasing resolution of global spectral models (Wedi et al., 2013), it is believed that the global spectral method is prohibitively expensive for very high resolution (Wedi, 2014).

A global (regional) spectral model performs FFT and FLT (FFT) in the zonal direction and the meridional direction, respectively. Because both transforms require all values in the corresponding directions, the parallelization of spectral method in a global (regional) model is usually conducted to exploit the horizontal domain decomposition only in the zonal direction and meridional directions for FFT and FLT (FFT), respectively (Barros et al., 1995; Kanamitsu et al., 2005). Owing to the horizontal domain decomposition in 


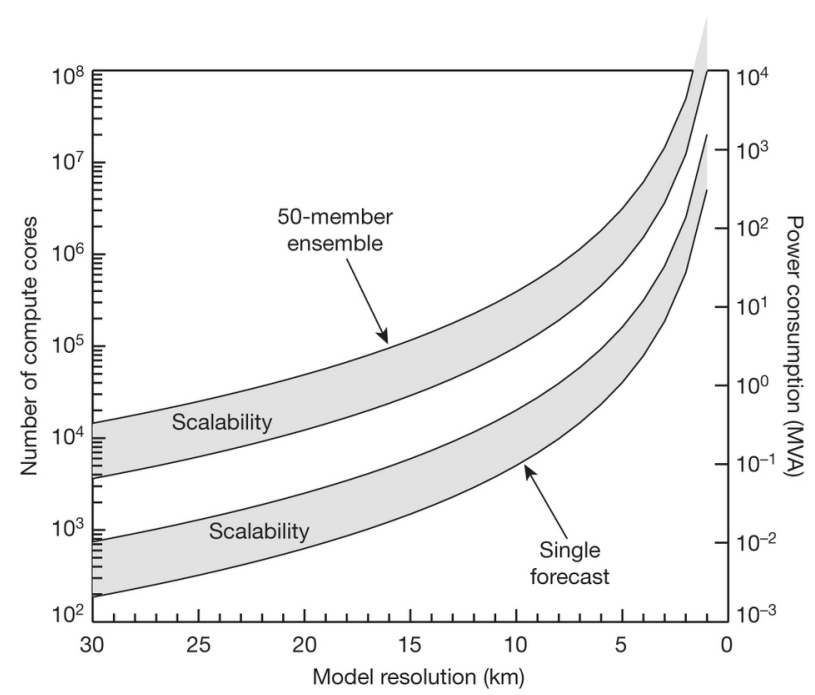

Figure 1. CPU and power requirements as a function of NWP model resolution, adapted from Bauer et al. (2015). The left and right $y$ axes are the number of cores and the power (in megavolt amps), respectively, required for a single 10-day model forecast (the lower shaded area including its bounds) and a 50-member ensemble forecast (the upper shaded area including its bounds) as a function of model resolution based on current model code and compute technology. The lower and upper bounds of each shaded area indicate perfect scaling and inefficient scaling, respectively.

a single horizontal direction for the parallelization of spectral transforms, there is a transposition between the spectral transforms in the zonal direction and meridional directions. MPI transposition is an all-to-all personalized communication which can cause significant congestion over an interconnect network when the number of MPI tasks and the amount of exchanged data are large, and this results in a severe communication delay. Bauer et al. (2015) estimated that a global NWP model with a $2 \mathrm{~km}$ horizontal resolution requires 1 million compute cores for a single 10-day forecast (Fig. 1). With 1 million compute cores, the performance and scalability of the MPI transposition become of paramount importance for a high-resolution global spectral model. Thus, evaluating and predicting the performance and scalability of MPI transposition at the exascale is one of the foremost subjects of this study.

The semi-Lagrangian (SL) method is a highly efficient technique for the transport of momentum, heat, and mass in the NWP model because of its unconditional stability which permits a long time step (Staniforth and Côté, 1991; Hortal, 2002). However, it is known that the MPI exchange of wide halo required for the interpolation at the departure point of high wind-speed particles near the boundary of the subdomain causes significant communication overhead as resolution increases towards the kilometre scale and the HPC systems move towards the exascale. This communication overhead could reduce the efficiency of the SL method; thus, modelling the performance and scalability of wide halo exchange at the exascale is essential and is another subject of this study.

With a consideration of the efficiency of the Legendre transform and the scalability of MPI transposition that may arise in the global spectral model on exascale HPC systems, a couple of global grid-point models have recently been developed (Lin, 2004; Satoh et al., 2008; Qaddouri and Lee, 2011; Skamarock et al., 2012; Dubos et al., 2015; Zangl et al., 2015; Smolarkiewicz et al., 2016). Since spherical harmonics are eigenfunctions of the Helmholtz operator, the semi-implicit (SI) method is usually adopted in order to implicitly handle the fast waves in the global spectral model to allow stable integration with a large time step (Robert et al., 1972; Hoskins and Simmons, 1975). However, for a grid-point model, the three-dimensional Helmholtz equation is usually solved using Krylov subspace methods such as the generalized conjugate residual (GCR) method (Eisenstat et al., 1983), and a global synchronization for the inner product in Krylov subspace methods may become the bottleneck at the exascale (Li et al., 2013; Sanan et al., 2016). As it is not clear whether the three-dimensional Helmholtz equation can be solved efficiently in a scalable manner, most of the aforementioned models use a horizontally explicit, vertically implicit (HEVI) scheme. The HEVI scheme typically requires some damping for numerical stability (Satoh et al., 2008; Skamarock et al., 2012; Zangl et al., 2015), and its time step is smaller than that of the SI method (Sandbach et al., 2015). Therefore, it is desirable to know whether the SI method is viable or even advantageous for very high-resolution gridpoint models running on exascale HPC systems. Thus, it is valuable to explore the performance and scalability of global synchronization in solving the three-dimensional Helmholtz equation using Krylov subspace methods; this forms the third subject of this study.

In this paper, we present the application of SST/macro 7.1, a coarse-grained parallel discrete event simulator, to investigate the communication performance and scalability of atmospheric models for future exascale supercomputers. The remainder of the paper is organized as follows. Section 2 introduces the simulation environment, the SST/macro simulator, and our optimizations for reducing the memory footprint and accelerating the simulations. Section 3 reviews three key MPI operations used in the atmospheric models. Section 4 presents and analyses the experimental results of the modelling communication of the atmospheric model using SST/macro. Finally, we summarize the conclusions and discuss future work in Sect. 5. 


\section{Simulation environment}

\subsection{Parallel discrete event simulation}

Modelling application performance on exascale HPC systems with millions of nodes and a complex interconnect network requires that the simulation can be decomposed into small tasks that efficiently run in parallel to overcome the problem of a large memory footprint and long simulation time. PDES is such an approach for exascale simulation. Each worker in PDES is a logical process (LP) that models a specific component such as a node, a switch, or an MPI process of the simulated MPI application. These LPs are mapped to the physical processing elements (PEs) that actually run the simulator. An event is an action such as sending an MPI message or executing a computation between consecutive communications. Each event has its start and stop times, so the events must be processed without violating their time ordering. To model the performance of an application, PDES captures time duration and advances the virtual time of the application by sending timestamped events between LPs.

PDES usually adopts conservative or optimistic parallelized strategies. The conservative approach maintains the time ordering of events by synchronization to guarantee that no early events arrive after the current event. Frequent synchronization is time-consuming so the efficiency of the conservative approach is highly dependent on the look-ahead time; a larger look-ahead time (that means less synchronization) allows a much greater parallelism. The optimistic approach allows LPs to run events at the risk of timeordering violations. Events must be rolled back when timeordering violations occurs. Rollback not only induces significant overhead but also requires extra storage for the event list. Rollback presents special challenges for online simulation, so SST/macro adopts a conservative approach (Wike and Kenny, 2014).

\subsection{SST/macro simulator}

Considering that the offline trace-driven simulation does not provide an easy way for extrapolating to future architectures, the online simulator SST/macro is selected here to model the communications of the atmospheric models for future exascale HPC systems. SST/macro is a coarse-grained parallel discrete event simulator which provides the best cost/accuracy trade-off simulation for large-scale distributed applications (Janssen et al., 2010). SST/macro is driven by either a trace file or a skeleton application. A skeleton application can be constructed from scratch or from an existing application manually or automatically by source-to-source translation tools. SST/macro intercepts the communications issued from the skeleton program to estimate their time rather than actually execute it by linking the skeleton application to the SST/macro library instead of the real MPI library. Since the purpose of this study is to investigate the performance and scalability of communications in an atmospheric model, we construct the communication-only skeleton program from scratch by identifying the key MPI operations taking place in the atmospheric models.

Congestion is a significant factor that affects the performance and scalability of MPI applications running on exascale HPC systems. SST/macro has three network models: the analytical model transfers the whole message over the network from point-to-point without packetizing and estimates the time delay $\Delta t$ predominantly based on the $\log \mathrm{P}$ approximation

$\Delta t=\alpha+\beta N$,

where $\alpha$ is the communication latency, $\beta$ is the inverse bandwidth in second per byte, and $N$ is the message size in bytes; the packet-level model PISCES (Packet-flow Interconnect Simulation for Congestion at Extreme Scale) divides the message into packets and transfers the packets individually; the flow-level model will be deprecated in the future. Compared to the SimGrid simulator, the packet-level model of SST/macro produces almost identical results (figure not shown). Acun et al. (2015) also found that the SST/macro online simulation is very similar to the TraceR simulation. Thus, we adopt the PISCES model with a cut-through mechanism (SNL, 2017) to better account for the congestion. SST/macro provides three abstract machine models for nodes: the AMM1 model is the simplest one which grants exclusive access to the memory; the AMM2 model allows multiple CPUs or NICs (network interface controllers) to share the memory bandwidth by defining the maximum memory bandwidth allocated for each component; the AMM3 model goes one further step to distinguish between the network link bandwidth and the switch bandwidth. In this paper, the AMM1 model with one single-core CPU per node is adopted since simulation of communications is the primary goal.

SST/macro provides several topologies of the interconnect network. In this study, three types of topologies (Fig. 2) commonly used in current supercomputers, and their configurations are investigated. Torus topology has been used in many supercomputers (Ajima et al., 2009). In the torus network, messages hop along each dimension using the shortest path routing from the source to the destination (Fig. 2a), and its bisection bandwidth typically increases with increasing dimension size of the torus topology. The practical implementation of the fattree topology is an upside-down tree that typically employs all uniform commodity switches to provide high bandwidth at higher levels by grouping corresponding switches of the same colour (Fig. 2c). Fattree topology is widely adopted by many supercomputers for its scalability and high path diversity (Leiserson, 1985); it usually uses a D-mod- $k$ routing algorithm (Zahavi et al., 2010) for desirable performance. A dragonfly network is a multi-level dense structure of which the high-radix routers are connected in a dense even all-to-all manner at each level (Kim et al., 2008). As shown in Fig. 2b, a typical dragonfly network consists 
(b)

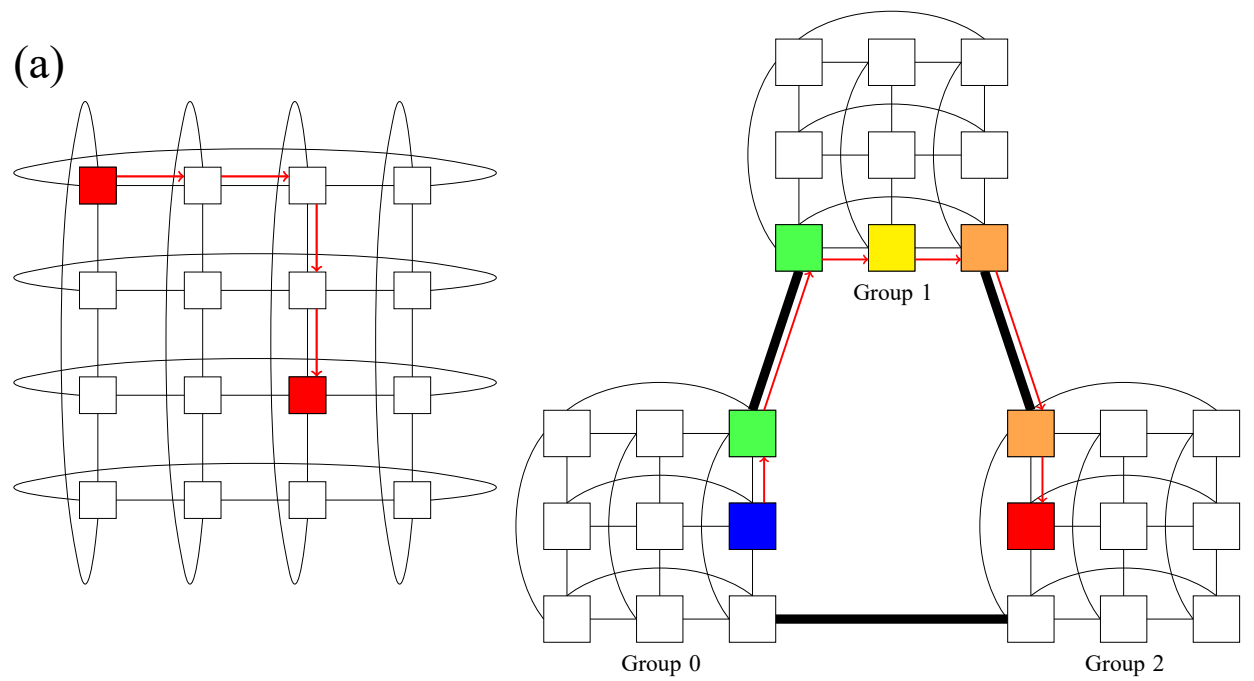

(c)

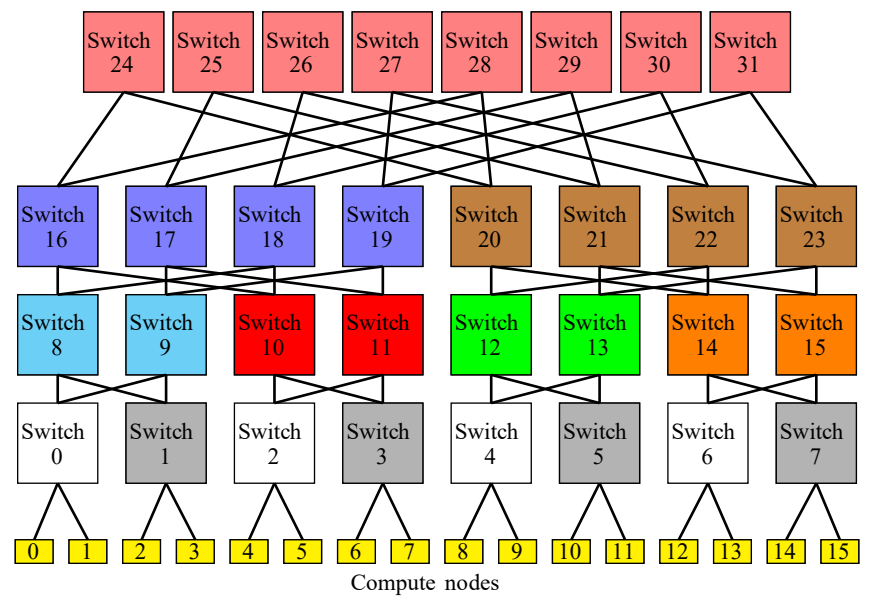

Figure 2. Topology illustration: (a), (b), and (c) are the torus, dragonfly, and fattree topologies, respectively. Adapted from SNL (2017).

of two levels: the routers at the first level are divided into groups and routers in each group form a two-dimension mesh of which each dimension is an all-to-all connected network; at the second level, the groups as virtual routers are connected in an all-to-all manner (Alverson et al., 2015). There are three available routing algorithms for dragonfly topology in SST/macro:

minimal transfers messages by the shortest path from the source to the destination. For example, messages travel from the blue router in group 0 to the red router in group 2 via the bottom-right corner in group 0 and the bottomleft corner in group 2 (Fig. 2b).

valiant randomly picks an intermediate router and then uses a minimal routing algorithm to transfer messages from the source to the intermediate router and from the intermediate router to the destination. For example, the arrow path from the blue router in group 0 to the red router in group 2 goes via the intermediate yellow node in group 1 in Fig. 2 b.

ugal checks the congestion and either switches to the valiant routing algorithm if congestion is too heavy or otherwise uses the minimal routing algorithm.

Table 1 summaries the network topology configurations used in this paper. The torus-M (torus-L) configuration is a 3D torus of $25 \times 25 \times 25(75 \times 25 \times 25)$ size. Fattree-M (fattreeL) configuration has four layers: the last layer consists of nodes while the other layers consist of switches with 25 (33) descendant ports per switch. We tested four configurations of dragonfly topology. The dragonfly-MM configuration has a medium group of a $25 \times 25$ mesh with 25 nodes per switch and a medium number $(=25)$ of groups. The dragonfly-SL configuration has a small group of a $25 \times 25$ mesh with five nodes per switch and a large number $(=125)$ of groups. The dragonfly-LS configuration has a large group of a $125 \times 125$ 
Table 1. Summary of the network topologies: the geometry of a torus topology specifies the size of each dimension; the first and second number in the geometry of a fattree topology are the number of layers and descendant ports per switch, respectively; the first two numbers and the last number in the geometry of a dragonfly topology indicate the group mesh size and the number of groups, respectively.

\begin{tabular}{lrrrr}
\hline Name & Geometry & Switches & Nodes per switch & Nodes \\
\hline Torus-M & $25,25,25$ & 15625 & 25 & 390625 \\
Fattree-M & 4,25 & 46875 & 25 & 390625 \\
Dragonfly-MM & $25,25,25$ & 15625 & 25 & 390625 \\
Dragonfly-SL & $25,25,125$ & 78125 & 5 & 390625 \\
Dragonfly-LS & $125,125,5$ & 78125 & 5 & 390625 \\
Torus-L & $75,25,25$ & 46875 & 25 & 1171875 \\
Fattree-L & 4,33 & 107811 & 33 & 1185921 \\
Dragonfly-ML & $25,25,75$ & 46875 & 25 & 1171875 \\
\hline
\end{tabular}

mesh with five nodes per switch and a small number $(=5)$ of groups. The dragonfly-ML configuration has a medium group of a $25 \times 25$ mesh with 25 nodes per switch and a large number $(=75)$ of groups. The fattree configuration has a significantly larger number of switches than other topologies for the same number of nodes and the same nodes per switch, which indicates that fattree is not cost- or energy-efficient. All the configurations with 390625 nodes are used for simulating transposition for the spectral transform method. Torus$\mathrm{L}$, fattree-L, and dragonfly-ML with more than 1 million nodes are used for the cases of halo exchange and allreduce communication since we cannot finish the simulation of transposition for the spectral transform method (multiple simultaneous all-to-all personalized communications) on such a large configuration within $24 \mathrm{~h}$ (see Sect. 3 for three key MPI communications in the atmospheric model).

\subsection{Reduce the memory footprint and accelerate the simulation}

Although SST/macro is a parallel discrete event simulator that can reduce the memory footprint per node, its parallel efficiency degrades if more cores are used. Even with an MPI transposition of $10^{5}$ processes, this all-to-all personalized communication has almost $10^{10}$ discrete events, which consumes a considerable amount of memory and takes a very long time for simulation. Furthermore, almost every MPI program has a set-up step to allocate memory for storing the set-up information such as the parameters and the domain decomposition of all processes, which each process must know in order to properly communicate with other processes. Therefore, it needs to broadcast the parameters to and synchronize with all processes before actual communications and computation. Even if the set-up information for a single process needs only $10^{2}$ bytes of memory, a simulation of $10^{5}$ processes MPI transposition will need $1 \mathrm{~TB}$ $\left(10^{2} \times 10^{5} \times 10^{5}=10^{12}\right.$ bytes $)$ of memory, which is not easily available on current computers if the simulator runs on a single node. In addition, the MPI operations in the set-up step are not only time-consuming but also affect subsequent communications. A common way to eliminate this effect is to iterate many times to obtain a robust estimation of communication time; however, one iteration is already very timeconsuming for simulation. To circumvent the issue of set-up steps, we use an external auxiliary program to create a shared memory segment on each node running SST/macro and initialize this memory with the set-up information of all the simulated MPI processes. Then, we modified SST/macro to create a global variable and attach the shared memory to this global variable; this method not only reduces the memory footprint and eliminates the side effect of communications in the set-up step, but it also avoids the problem of filling up the memory address space if each simulated process attaches to the shared memory.

A large-scale application needs a large amount of memory for computation; and in some cases, such as a spectral model, the whole memory for computation is exchanged between all the processes. Even when computation is not considered, a large amount of memory for the message buffers is usually required for MPI communications. Fortunately, the simulator only needs message size, the source/destination, and the message tag to model the communication; thus, it is not necessary to allocate actual memory. Since SST/macro can operate with null buffers, the message buffer is set to null in the skeleton application, which significantly reduces the size of memory required by the simulation of communication of the high-resolution atmospheric model.

\section{Key MPI operations in atmospheric models}

\subsection{Transposition for the spectral transform method}

A global spectral model generally uses a spherical-harmonics transform on the horizontal with triangular truncation. The backward spherical-harmonics transform is

$f(\theta, \lambda)=\sum_{m=-M}^{M}\left(e^{i m \lambda} \sum_{n=|m|}^{M} f_{n}^{m} P_{n}^{m}(\cos \theta)\right)$, 
where $\theta$ and $\lambda$ are the colatitude and longitude, $f_{n}^{m}$ is the spectral coefficients of the field $f$, and $P_{n}^{m}$ is the associated Legendre polynomials of degree $m$ and order $n$. Moreover, the forward spherical-harmonics transform is

$$
f_{n}^{m}=\frac{1}{2} \int_{-1}^{1}\left(P_{n}^{m}(\cos \theta) \frac{1}{2 \pi} \int_{0}^{2 \pi} f(\theta, \lambda) e^{-i m \lambda} d \lambda\right) d \cos \theta
$$

In Eq. (2), the backward Legendre transform of each $m$ can be computed independently; then, the same applies to the backward Fourier transform of each $\theta$. Similar in Eq. (3), the forward Fourier transform of each $\theta$ can be computed independently; then, the same applies to the forward Legendre transform of each $m$. This leads to a natural way to parallelize the spectral transforms. If we start with the grid-point space (Fig. 3a), which is decomposed by $c x / c y$ cores in the $x / y$ direction, $c y$-simultaneous $x z$ slab MPI transpositions lead to the partition (Fig. 3b) with $c y / c x$ cores in the $y / z$ direction, and a spectral transform such as a forward FFT can be performed in parallel since data with regard to $\lambda$ are local to each core. Then, $c x$-simultaneous $x y$ slab MPI transpositions lead to the partition (Fig. 3c) with $c y / c x$ cores in the $x / z$ direction, and a spectral transform such as a forward FLT can be computed in parallel because data with regard to $\theta$ are now local to each core. Finally, $c y$-simultaneous $y z$ slab MPI transpositions lead to the spectral space (Fig. 3d) with $c y / c x$ cores in the $x / y$ direction, where the semi-implicit scheme can be easily computed because spectral coefficients belonging to the same column are now local to the same core. The backward transform is similar. It is of paramount importance that the partition of the four stages described in Fig. 3 must be consistent so that multiple slab MPI transpositions can be conducted simultaneously, which significantly reduces the communication time of MPI transpositions from one stage to another. It is worth noting that the number of grid points in one direction is not always a multiple of the number of cores in the corresponding direction; thus, the partition shown in Fig. 3 can use as many as possible compute cores without any limit on $c x$ or $c y$ provided $c x \times c y=$ ncpu and $c x$ or $c y$ is not greater than the number of grid points in the corresponding direction. It is generally believed that the MPI transpositions from one stage to another pose a great challenge to the scalability of spectral models because each slab MPI transposition is an all-to-all personalized communication which is the most complex and time-consuming all-to-all communication.

There are different algorithms for all-to-all personalized communication. Table 2 lists the three algorithms for allto-all personalized communication, whose performance and scalability are investigated in this study. Algorithm ring $k$ is our proposal algorithm for all-to-all personalized communication which is a generalized ring alltoallv algorithm. In algorithm ring $k$, each process communicates with $2 \times k$ processes to reduce the stages of communications and make ef-
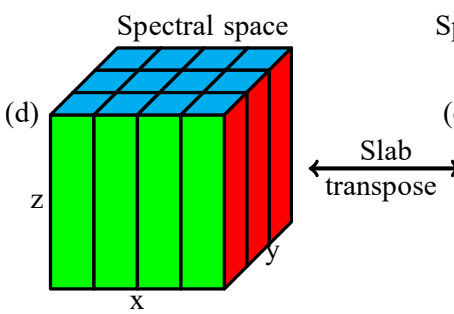

Spectral transform in y direction

(c)

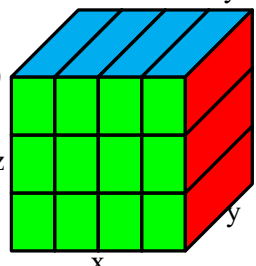

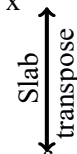

(a)
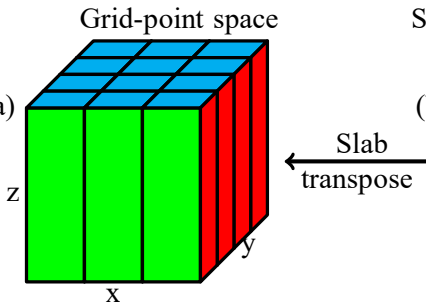

Spectral transform in $\mathrm{x}$ direction

(b)

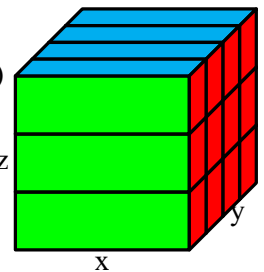

Figure 3. Parallel scheme of regional spectral model: (a) 2-D decomposition of 3-D grid field with $c x / c y$ cores in the $x / y$ direction, (b) 2-D decomposition of 3-D grid field with $c y / c x$ cores in the $y / z$ direction, (c) 2-D decomposition of 3-D grid field with $c y / c x$ cores in the $x / z$ direction, and (d) 2-D decomposition of 3-D grid field with $c y / c x$ cores in the $x / y$ direction. Transposition between (a) and (b) can be conducted by $c y$-independent $x z$ slab MPI transpositions, transposition between (b) and (c) can be conducted by $c x$-independent $x y$ slab MPI transpositions, and transposition between (c) and (d) can be conducted by $c y$-independent $y z$ slab MPI transpositions.

ficient use of the available bandwidth and thus reduces the total communication time.

\subsection{Halo exchange for semi-Lagrangian method}

The SL method solves the following transport equation:

$\frac{\mathrm{D} \phi}{\mathrm{D} t}=\frac{\partial \phi}{\partial t}+u \frac{\partial \phi}{\partial x}+v \frac{\partial \phi}{\partial y}+w \frac{\partial \phi}{\partial z}=0$,

where the scalar field $\phi$ is advected by the 3-D wind $\mathbf{V}=$ $(u, v, w)$. In the SL method, the grid-point value of the scalar field $\phi$ at next time step $t+\Delta t$ can be found by integrating Eq. (4) along the trajectory of the fluid parcel (Staniforth and Côté, 1991; Hortal, 2002):

$\int_{t}^{t+\Delta t} \frac{\mathrm{D} \phi}{\mathrm{D} t} d t=0 \rightarrow \phi^{t+\Delta t}=\phi_{d}^{t}$

where $\phi^{t+\Delta t}$ is the value of the fluid parcel $\phi$ arriving at any grid point at $t+\Delta t$ and $\phi_{d}^{t}$ is the value of the same fluid parcel at its departure point $d$ and departure time $t$. This means that the value of the scalar field $\phi$ at any grid point at $t+\Delta t$ is equal to its value at the departure point $d$ and the departure time $t$. The departure point $d$ usually does not coincide 
Table 2. Three algorithms for all-to-all personalized communication.

\begin{tabular}{|c|c|c|}
\hline Name & Description & Stages \\
\hline Burst & $\begin{array}{l}\text { Each process communicates with all other processes simultaneously by posting all non-block send and } \\
\text { receive operations simultaneously. The burst messages cause significant congestion in the network. This } \\
\text { algorithm is equivalent to the algorithm ring } k \text { when } k=n-1 \text {. }\end{array}$ & 1 \\
\hline Bruck & $\begin{array}{l}\text { This algorithm is better for small message and a large latency since it has only }\left\lceil\log _{2}(n)\right\rceil \text { stages of com- } \\
\text { munications (Thakur et al., 2005). For the } k \text { th stage, each process sends the messages whose destination } \\
\text { process ID has one at the } k \text { th bit (begin at least significant bit) to process } i+2^{k} \text {. }\end{array}$ & $\left\lceil\log _{2}(n)\right\rceil$ \\
\hline Ring $k$ & $\begin{array}{l}\text { In the first stage, process } i \text { sends to } i+1, \cdots, i+k \text { and receives from } i-1, \cdots, i-k \text { in a ring way } \\
\text { (black arrows in Fig. 4a); in the second stage, process } i \text { sends to } i+1+k, \cdots, i+2 k \text { and receives from } \\
i-1-k, \cdots, i-2 k \text { in a ring way (blue arrows in Fig. 4a); this continues until all partners have been } \\
\text { communicated with. This algorithm is a generalization of the ring algorithm and efficiently uses the } \\
\text { available bandwidth by proper selection of radix } k \text {. }\end{array}$ & $\left\lceil\frac{n-1}{k}\right\rceil$ \\
\hline
\end{tabular}

with any grid point, so the value of $\phi_{d}^{t}$ is obtained by interpolation using the surrounding grid-point values $\phi^{t}$ at time $t$. The departure point $d$ is determined by iteratively solving the trajectory equation (Staniforth and Côté, 1991; Hortal, 2002)

$$
\frac{\mathrm{D} \boldsymbol{r}}{\mathrm{D} t}=\mathbf{V}(\boldsymbol{r}, t) \rightarrow \boldsymbol{r}^{t+\Delta}-\boldsymbol{r}_{d}^{t}=\int_{t}^{t+\Delta t} \mathbf{V}(\boldsymbol{r}, t) d t
$$

where $\boldsymbol{r}^{t+\Delta t}$ and $\boldsymbol{r}_{d}^{t}$ are the position of the arrival and the departure point, respectively. From Eq. (6), it is obvious that the departure point is far from its arrival point if the wind speed is large. Thus, the departure point of one fluid parcel at the boundary of the subdomain of an MPI task is far from its boundary if the wind speed is large and the wind blows from the outside. To facilitate the calculation of the departure point and its interpolation, MPI parallelization adopts a "maximum wind" halo approach so that the halo is sufficiently large for each MPI task to perform its SL calculations in parallel after exchanging the halo. This "maximum wind" halo is named "wide halo" since its width is significantly larger than that of the thin halo of finite difference methods whose stencils have compact support. With numerous MPI tasks, the width of a wide halo may be larger than the subdomain size of its direct neighbour, which implies that the process needs to exchange the halo with its neighbours and its neighbours' neighbours, which may result in a significant communication overhead which counteracts the efficiency of the favourite SL method and poses a great challenge to the scalability of the SL method.

Figure $4 \mathrm{~b}$ demonstrates the halo exchange algorithm adopted in this paper. First, the algorithm posts the MPI nonblock send and receive operations 1-4 simultaneously for the $x$-direction sweep. After the $x$-direction sweep, a $y$-direction sweep is performed in a similar way but the length of halo is extended to include the left and right halo in the $x$ direction so that the four corners are exchanged properly. This algorithm needs two stages of communications but is simple to implement, especially for the wide halo exchange, owing to its fixed regular communication pattern (Fig. 9d). In Fig. 9d, the pixels (purplish colour) tightly attached to the diagonal are due to the exchange in the $x$ direction; the pixels of the same colour but off-diagonal are because of the periodicity in $x$ direction; the pixels (reddish orange colour) off-diagonal are due to the exchange in the $y$ direction; and the pixels of the same colour but far off the diagonal are because of the periodicity in the $y$ direction. This algorithm also applies to the thin halo exchange for finite difference methods, which is extensively used in the grid-point models. The study emphasizes the wide halo exchange, but the thin halo exchange is also investigated for comparison (see the red line in Fig. 9a).

\subsection{Allreduce in Krylov subspace methods for the semi-implicit method}

The three-dimensional SI method leads to a large linear system which can be solved by Krylov subspace methods:

$\mathbf{A} \boldsymbol{x}=\boldsymbol{b}$,

where $\mathbf{A}$ is a non-symmetric sparse matrix. Krylov subspace methods find the approximation $\boldsymbol{x}$ iteratively in a $k$ dimensional Krylov subspace:

$\mathcal{K}=\operatorname{span}\left(\boldsymbol{r}, \mathbf{A} \boldsymbol{r}, \mathbf{A}^{2} \boldsymbol{r}, \cdots, \mathbf{A}^{k-1} \boldsymbol{r}\right)$,

where $\boldsymbol{r}=\boldsymbol{b}-\mathbf{A} \boldsymbol{x}$. To accelerate the convergence, preconditioning is generally used:

$\mathbf{M}^{-1} \mathbf{A} \boldsymbol{x}=\mathbf{M}^{-1} \boldsymbol{b}$

where $\mathbf{M}$ approximates $\mathbf{A}$ well so that $\mathbf{M}^{-1} \mathbf{A}$ be conditioned better than $\mathbf{A}$ and $\mathbf{M}^{-1}$ can be computed cheaply. The GCR method is a Krylov subspace method of easy implementation and can be used with variable preconditioners. Algorithm 1 of GCR shows that there are two allreduce operations using the sum operation for the inner product in each 
iteration; thus, it has $2 \mathrm{~N}$ allreduce operations if the GCR iterative solver reaches convergence in $N$ iterations. Allreduce is an all-to-all communication and becomes expensive when the number of iterations becomes larger in GCR solver with numerous MPI processes.

Figure $4 \mathrm{c}$ demonstrates the recursive- $k$ algorithm for the allreduce operation, which is a generalization of the recursive doubling algorithm. The radix $k$ is the number of processes in a group for the recursive- $k$ algorithm. Let $p=\left\lfloor\log _{k}(\mathrm{ncpu})\right\rfloor$. This algorithm has $p$ stages of communications if the number of processes is a power of radix $k$; otherwise it has two extra stages of communications in the beginning and ending of the algorithm. The following description of the recursive- $k$ algorithm applies to any number of processes; that is, the first and last stage are not necessary when the number of processes is a power of radix $k$. In the first stage with stage ID $j=0$ (the first row in Fig. 4c), each remaining process whose ID $i \notin\left[0, k^{p}-1\right]$ sends its data to process $i-\left(\mathrm{ncpu}-k^{p}\right)$ for the reduce operation. For the stage of stage ID $j \in[1, p]$ (rows between the first row and second last row in Fig. 4c), all the processes with the same value of $\bmod \left(i, k^{j-1}\right)$ form a list of processes in ascending order of $i$, where $i \in\left[0, k^{p}-1\right]$ is the process ID and $\bmod \left(i, k^{j-1}\right)$ is the remainder of $i$ divided by $k^{j-1}$. Then, every $k$ processes in this ordered list form a group of processes; i.e., the first $k$ processes form the first group, the second $k$ processes form the second group, etc. Each group of processes performs its allreduce operation independently. In the final stage with stage ID $j=1+p$ (the second last row in Fig. 4c), each process whose ID $i \notin\left[0, k^{p}-1\right]$ receives its final result from process $i-\left(\mathrm{ncpu}-k^{p}\right)$. The recursive- $k$ algorithm uses large radix $k$ to reduce the stages of communications and the overall communication time.

\section{Experimental results}

\subsection{Experiment design}

In the next decade, it is estimated that the resolution of global NWP models will approach the kilometre scale and the HPC will move towards the exascale. What would the performance of a global NWP model with a very high resolution on exascale HPC be? In this paper, we are especially interested in the strong scaling of an atmospheric model, that is, how does the atmospheric model with fixed resolution (such as the one presented in Table 3) behave as the number of processes increases? In this study, these strong scalings of the three key MPI operations in the atmospheric model are assessed for $10^{2}, 2 \times 10^{2}, \cdots, 9 \times 10^{2}, 10^{3}, 2 \times 10^{3}, \cdots, 9 \times 10^{3}, 10^{4}, 2 \times$ $10^{4}, \cdots, 9 \times 10^{4}, 10^{5}, 2 \times 10^{5}, \cdots, 9 \times 10^{5}, 10^{6}$ MPI tasks; but the maximum number of processes is $2 \times 10^{5}$ for the MPI transposition owing to the hard time limitation in our cluster. Table 3 presents a summary of the three-dimensional grid for assessing the communication of the kilometre-scale at-
Table 3. A three-dimensional grid for assessing the communication of the atmospheric model. $\Delta x$ and $\Delta y$ are given as if this grid is a uniform global longitude-latitude grid. In fact, this grid resembles the grid of a regional spectral atmospheric model or the uniform longitude-latitude grid used by some global models.

\begin{tabular}{ccc|ccc}
\hline$n x$ & $n y$ & $n z$ & $\Delta x$ & $\Delta y$ & Grid points \\
\hline 28800 & 14400 & 256 & $0.0125^{\circ}$ & $0.0125^{\circ}$ & $>100$ billion \\
\hline \multicolumn{3}{c|}{ Memory size } & \multicolumn{3}{c}{ Max. processes } \\
\hline$>$ 800 GB per double field & \multicolumn{3}{c}{3686400 for a 2-D partition } \\
\hline
\end{tabular}

mospheric model. The number of grid points of this grid is beyond 100 billion, and one field of double-precision variables for this grid requires more than 800 gigabytes of memory. Only with such a large grid is it possible to perform a 2-D domain decomposition for a spectral model with more than 1 million processes so that modelling the communication of the atmospheric model at exascale HPC becomes possible.

Besides the topology and its configuration, the routing algorithm, and the collective MPI algorithm; the bandwidth and the latency of the interconnect network of an HPC system have a great impact on the performance of communications. First, we simulate the transposition for the spectral transform method in the simulator for three topologies (torus$\mathrm{M}$, fattree-M, and dragonfly-MM in Table 1), three configurations of dragonfly topology (dragonfly-MM, dragonflySL, and dragonfly-LS in Table 1), three routing algorithms (minimal, valiant, and ugal), and three alltoallv algorithms (Table 2). In addition, we compare the simulations of the transposition for the spectral transform method between four interconnect bandwidths $\left(10^{0}, 10^{1}, 10^{2}\right.$, and $\left.10^{3} \mathrm{~GB} \mathrm{~s}^{-1}\right)$ and between four interconnect latencies $\left(10^{1}, 10^{2}, 10^{3}\right.$, and $\left.10^{4} \mathrm{~ns}\right)$. After a thorough investigation of the transposition for the spectral transform method, we test the halo exchange for the SL method with different halo widths $(3,10,20$, and 30 grid points), three topologies (torus-L, fattree-L, and dragonfly-ML in Table 1), and three routing algorithms (minimal, valiant, and ugal). Finally, the allreduce operation in Krylov subspace methods for the SI method is evaluated on different topologies (torus-L, fattree-M, and dragonfly-ML in Table 1), and the statistics of the optimal radix of recursive- $k$ algorithms for allreduce operations are presented.

\subsection{Transposition for the spectral transform method}

Figure 5a shows that the communication times for the burst, bruck, ring-1, and ring- 4 algorithms decrease as the number of MPI processes increases. The ring- 1 and ring- 4 algorithms are almost identical for fewer than $5 \times 10^{4}$ MPI processes, but ring 4 performs better than ring 1 for more than $10^{5}$ MPI processes. The burst and bruck algorithms perform worse than the ring- $k$ algorithm. The SST/macro simulator cannot simulate the burst algorithm for more than $2 \times 10^{4}$ MPI pro- 


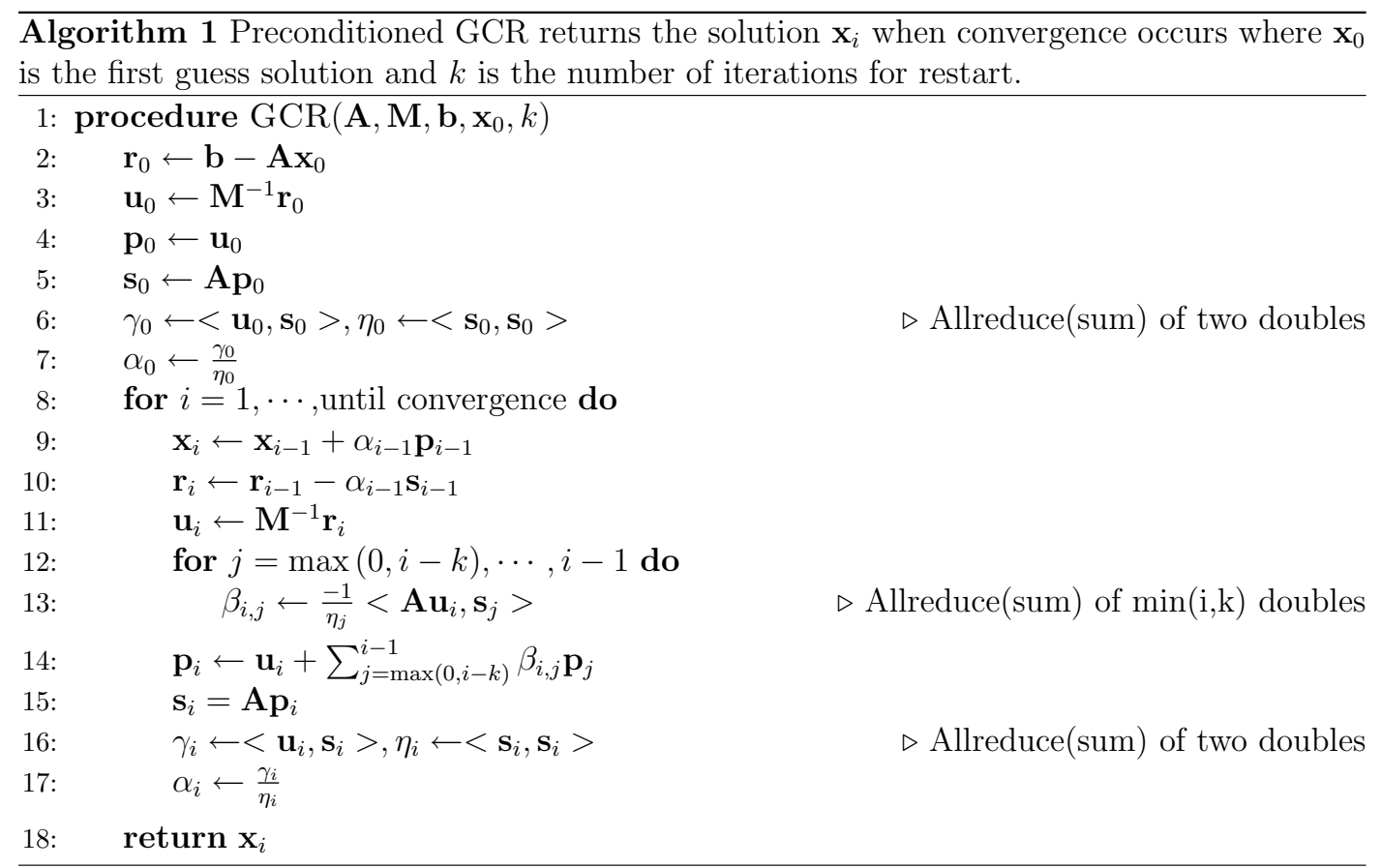

cesses because the burst messages result in huge events and a large memory footprint. The communication time of the bruck algorithm is significantly larger than that of the ring- $k$ algorithm for fewer than $10^{5}$ MPI processes; however, for a greater number of processes, it is better than the ring-1 algorithm since the bruck algorithm is targeted for small messages, and the more processes, the smaller the message for a fixed-size problem. The performance of these alltoallv algorithms is confirmed by actually running the skeleton program of transposition for the spectral transform method with $10^{4}$ MPI processes on the research cluster (Beaufix) of Météo France, which shows that the ring-4 algorithm is even better than the INTEL native MPI_Alltoallv function (Fig. 6).

The differences in the communication times of the transpositions between the topology torus- $\mathrm{M}$, fattree-M, and dragonfly-MM can be an order of magnitude (Fig. 5b). Messages have to travel a long distance in the topology torus$\mathrm{M}$ which is a 3-D torus, so its communication time is the largest. The best performance of the topology fattree-M can be attributed to its non-blocking D-mod- $k$ routing algorithm, but its communication time gradually increases as the number of MPI processes increases beyond $10^{4}$. The performance of topology dragonfly-MM is between that of torus-M and fattree-M (Fig. 5b); it can achieve a better performance by tuning the configuration of the dragonfly topology (Fig. 5c). By comparing Fig. 5b and c, we can see that the topologies of dragonfly-SL and dragonfly-LS are still not as good as the fattree-M, but their performance is very close to that of fattree-M and they lose less scalability than fattree-M for more than $5 \times 10^{4}$ MPI processes.
The differences in communication time of the transpositions between the routing algorithms of minimal, valiant, and ugal are also an order of magnitude (Fig. 5d), which indicates that the impact of routing algorithm on communication is significant. The valiant routing algorithm performs the best, but the communication time begins to increase when the number of MPI processes is larger than $3 \times 10^{4}$. The ugal routing algorithm performs the worst, and the performance of the minimal routing algorithm is in between that of the valiant and ugal routing algorithms. The valiant routing algorithm has the longest path for messages from the source to the destination with a randomly chosen intermediate node; thus, theoretically, its communication time is larger. By contrast, the minimal routing algorithm that moves the messages using the shortest path from the source to the destination has the smallest communication time. The congestion between processes in Fig. 7 shows that the valiant routing algorithm for the dragonfly-MM topology (Fig. 7b) and the minimal routing algorithm for the dragonfly-SL topology (Fig. 7d) are less congested and have a more uniform congestion, the minimal routing algorithm for the dragonflyMM topology is moderately congested, but its congestion is not uniform (Fig. 7a), and the congestion of the ugal routing algorithm for the dragonfly-MM topology is large and highly non-uniform (Fig. 7c). These congestions in Fig. 7 are consistent with the communication times in Fig. $5 \mathrm{c}$ and d, that is, the more uniform the congestion, the lower the communication time because the latter is determined by the longest delay event and uniform congestion can avoid the hotspots of the congestion with the longest delay event. Figure 8 con- 
(b)
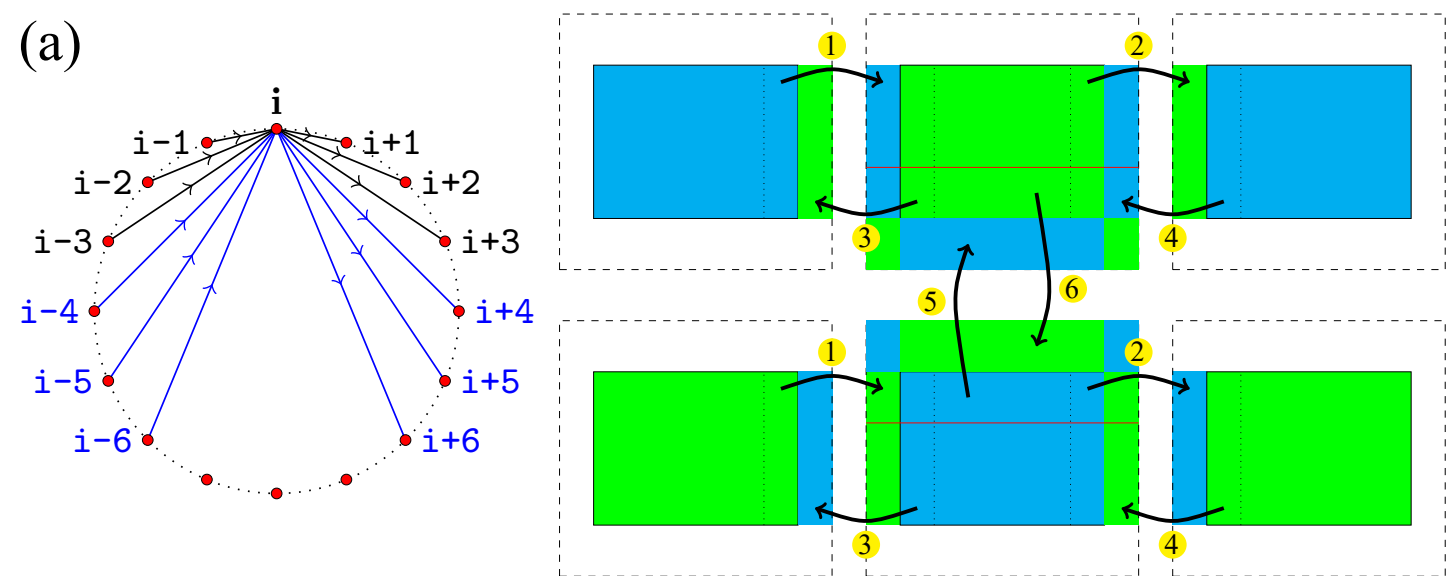

(c)

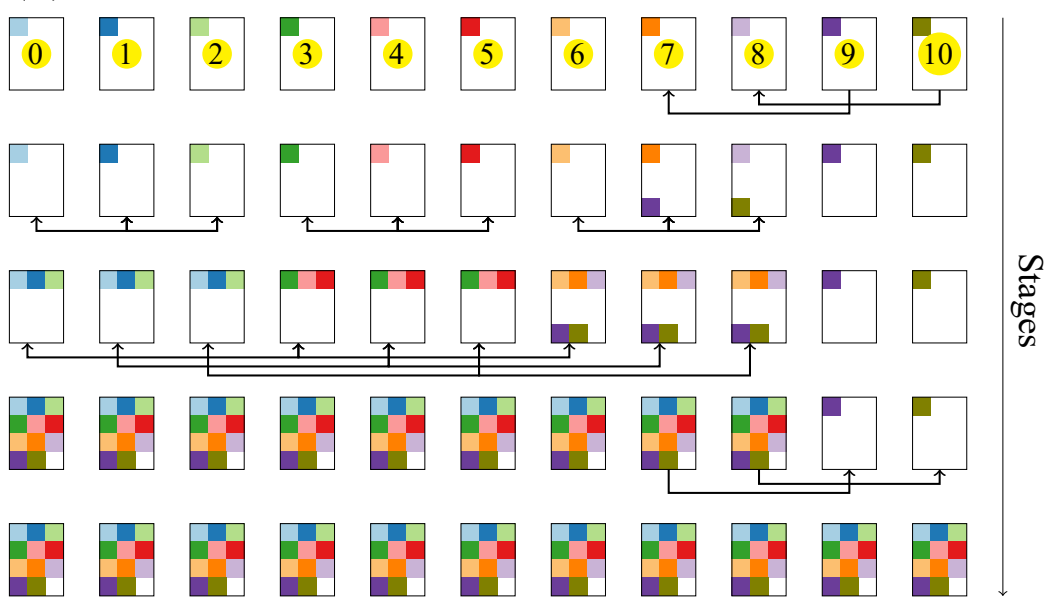

Figure 4. Algorithms for three key MPI operations: (a) is the ring- $k$ algorithm with $k$ radix for all-to-all personalized communication generalized from ring alltoallv algorithm, (b) is the halo exchange algorithm, and (c) is the recursive- $k$ algorithm with $k$ radix generalized from the recursive doubling algorithm.

firms this that a high percentage of delay events has a delay time of fewer than $30 \mu$ s using the valiant routing algorithm for the dragonfly-MM topology and the minimal routing algorithm for the dragonfly-SL topology; however, the minimal routing algorithm for the dragonfly-MM topology has a significant percentage of events that delays by more than $50 \mu$ s, especially there are a large number of events delayed by more than $100 \mu$ s using the ugal routing algorithm for the dragonfly-MM topology. Thus, the configuration of the interconnect network and the design of its routing algorithm should make the congestion as uniform as possible if congestion is inevitable.

Although the communication time with a bandwidth of $10^{0} \mathrm{~GB} \mathrm{~s}^{-1}$ is apparently separated from those with bandwidths of $10^{1}, 10^{2}$, and $10^{3} \mathrm{~GB} \mathrm{~s}^{-1}$, the curves describing the communication times with bandwidths of $10^{1}, 10^{2}$, and $10^{3} \mathrm{~GB} \mathrm{~s}^{-1}$ overlap (Fig. 5e). The communication times with latencies of $10^{1}$ and $10^{2} \mathrm{~ns}$ are almost identical; that with a latency of $10^{3}\left(10^{4}\right) \mathrm{ns}$ is slightly (apparently) different from those with latencies of $10^{1}$ and $10^{2} \mathrm{~ns}$ (Fig. 5f). Equation (1) indicates that the communication time stops decreasing only when $\alpha(\beta)$ approaches zero and $\beta(\alpha)$ is constant given a fixed message size. Neither $\alpha$ in Fig. 5e nor $\beta$ in Fig. 5f approaches zero, but the communication time stops decreasing. The inability of the analytical model (1) to explain this suggests that other dominant factors such as congestion contribute to the communication time. Latency is the amount of time required to travel the path from one location to another. Bandwidth determines how much data per second can be moved in parallel along that path and limits the maximum number of packets travelling in parallel. Because both $\alpha$ and $\beta$ are greater than zero, congestion occurs when data arrive at a network interface at a rate faster than the media can service; when this occurs, packets must be placed in a queue to wait until earlier packets have been serviced. The longer the wait, the longer the delay and communication time. Fig- 


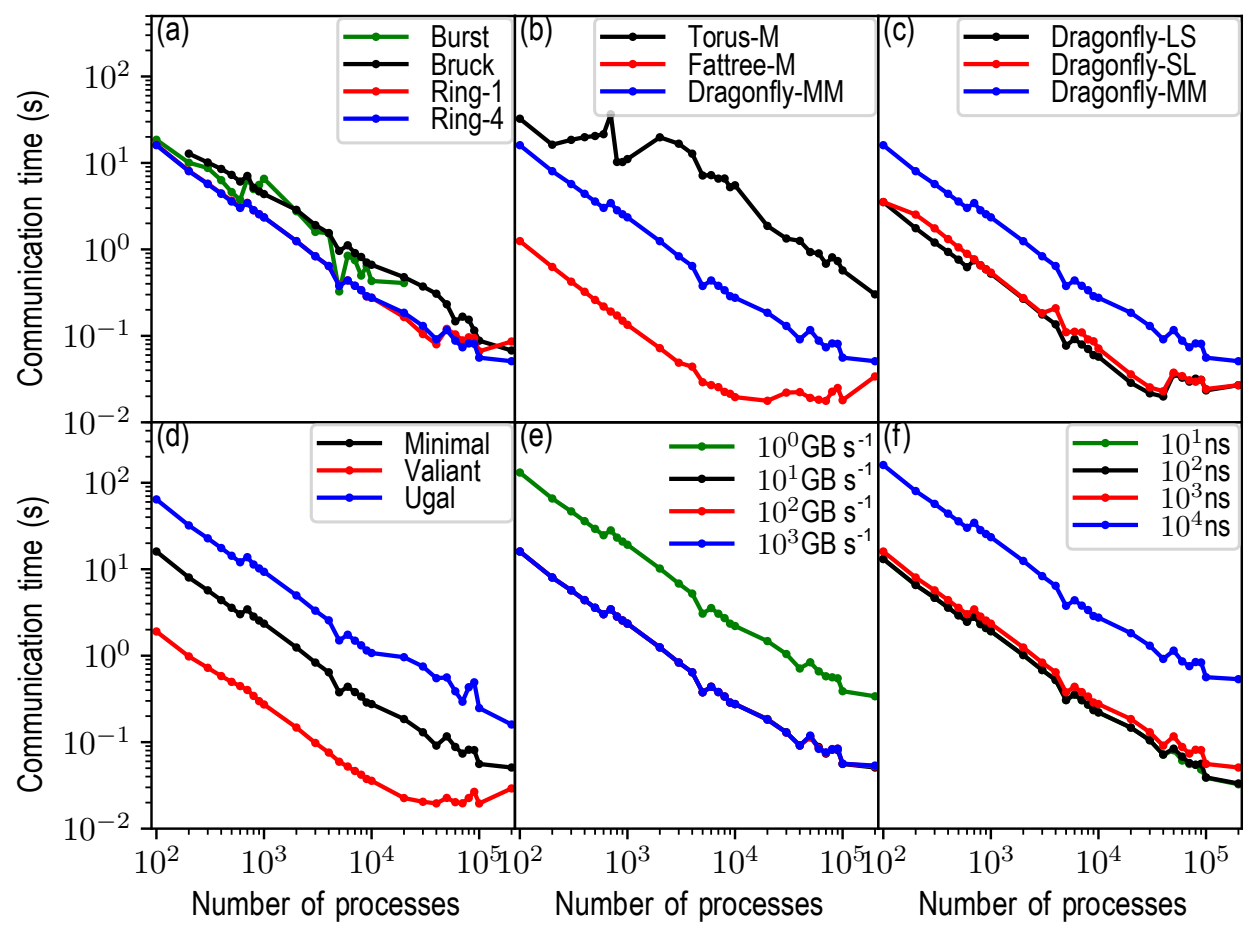

Figure 5. Communication times of transposition for (a) alltoallv algorithms, (b) topologies, (c) configurations of the dragonfly topology, (d) routing algorithms for the dragonfly topology, (e) bandwidth, and (f) latency. The circle markers indicate the numbers of processes of the corresponding simulations.

ure $8 \mathrm{~b}$ and $\mathrm{c}$ show the distributions of the delay caused by congestion for different bandwidths and different latencies, respectively. In Fig. 8b, the distributions of the delay for bandwidths of $10^{1}, 10^{2}$, and $10^{3} \mathrm{~GB} \mathrm{~s}^{-1}$ are almost identical, which explains their overlapped communication times in Fig. 5e; and the distribution of the delay for a bandwidth of $10^{0} \mathrm{~GB} \mathrm{~s}^{-1}$ is distinct from the rest since near $20 \%$ of events are delayed by fewer than $10 \mu$ s but a significant percentage of events are delayed more than $100 \mu$ s, which accounts for its largest communication time in Fig. 5e. In Fig. 8c, the distributions of the delay for latencies of $10^{1}$ and $10^{2} \mathrm{~ns}$ are the same; the distributions of the delay for a latency of $10^{3} \mathrm{~ns}$ is slightly different from the formers; but the distributions of the delay for a latency of $10^{4} \mathrm{~ns}$ has a large percentage of events in the right tail, which resulted in the longest communication time; these are consistent with their communication times in Fig. 5f.

In summary, the alltoallv algorithm, the topology and its configuration, the routing algorithm, the bandwidth, and the latency have great impacts on the communication time of transpositions. In addition, the communication time of transpositions decreases as the number of MPI processes increases in most cases; however, this strong scalability is not applicable for the fattree-M topology (the red line in Fig. 5b), the dragonfly-SL and dragonfly-LS topologies (red and black lines in Fig. 5c), and the valiant routing algorithm (the red line in Fig. 5d) when the number of MPI processes is large.

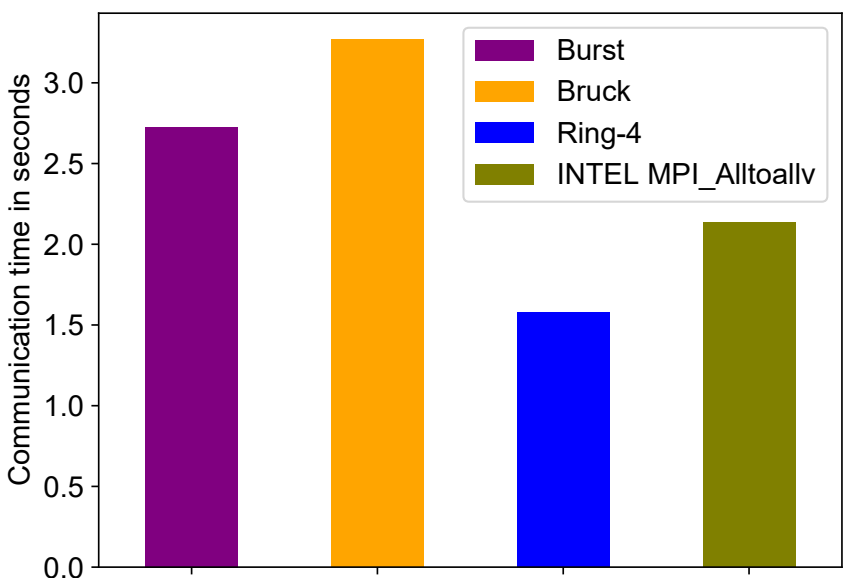

Figure 6. Actual communication time of transposition for the spectral transform method with $10^{4}$ MPI processes run on the Beaufix cluster in Météo France.

Thus, the topology of the interconnect network and its routing algorithm have a great impact on the scalability of transpositions for the spectral transform method. Since the transposition for the spectral transform method is a multiple simultaneous all-to-all personalized communication, congestion has a great impact on its performance. 


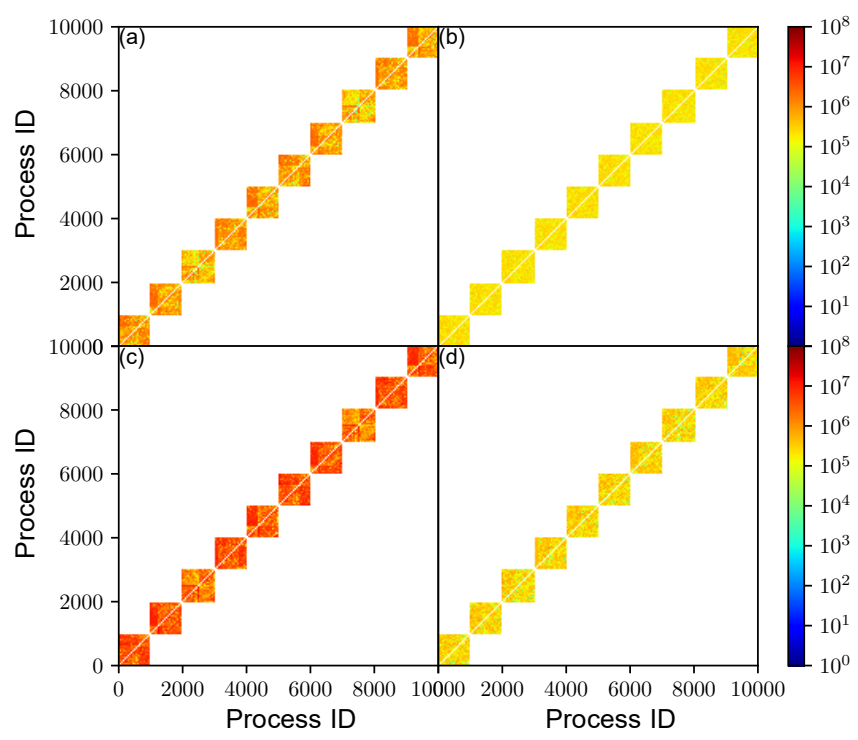

Figure 7. Congestion of transposition using (a) minimal routing algorithm for the dragonfly-MM topology, (b) valiant routing algorithm for the dragonfly-MM topology, (c) ugal routing algorithm for the dragonfly-MM topology, and (d) minimal routing algorithm for the dragonfly-SL topology.

\subsection{Halo exchange for the semi-Lagrangian method}

The most common application of the wide halo exchange is the SL method. For the resolution of $0.0125^{\circ}$ in Table 3 and a time step of $30 \mathrm{~s}$, the departure is approximately five grid points away from its arrival if the maximum wind speed is $200 \mathrm{~m} \mathrm{~s}^{-1}$; therefore, the width of the halo is at least seven grid points using the ECMWF quasi-cubic scheme (Ritchie, 1995); there are more grid points if a higher-order scheme such as the SLICE-3D (Semi-Lagrangian Inherently Conserving and Efficient (SLICE) in three dimensions) (Zerroukat and Allen, 2012) is used. In Fig. 9a, the communication time of the halo exchange decreases more slowly with an increasing number of processes than that of transposition for the spectral transform method. This is because the message size decreases more slowly than that of transposition owing to the fixed width of the halo (figure not shown). If the communication time of the transposition (halo exchange) continues its decreasing (increasing) trend in Fig. 9a, they meet at certain number of MPI processes; then, the communication time of the halo exchange is larger than that of the transposition. In addition, it can be seen that the wider the halo, the longer the communication time. The halo exchange of a thin halo of three grid points, such as for the sixth-order central difference $F_{i}^{\prime}=\frac{-F_{i-3}+9 F_{i-2}-45 F_{i-1}+45 F_{i+1}-9 F_{i+2}+F_{i+3}}{60 \Delta}$ (the red line in Fig. 9a), is significantly faster than that of wide halo for the SL method (green and blue lines in Fig. 9a). Thus, the efficiency of the SL method is counteracted by the overhead of the wide halo exchange where the width of the halo is determined by the maximum wind speed. Wide

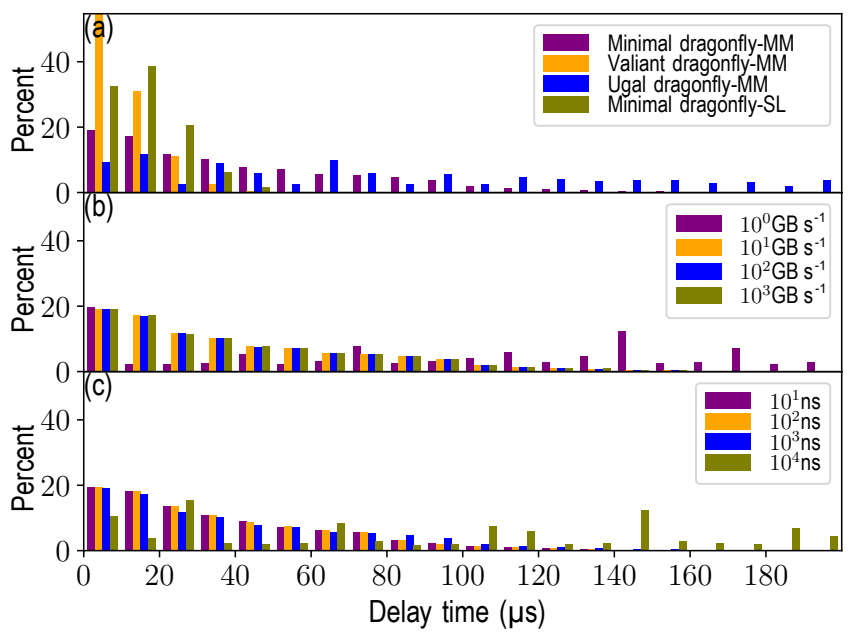

Figure 8. Distribution of delayed events of transposition for the spectral transform method with $10^{4}$ MPI processes using (a) different routing algorithms and topology configurations, (b) different bandwidths, and (c) different latencies, simulated by SST/macro.

halo exchange for the SL method is expensive at the exascale, especially for the atmospheric chemistry models where a large number of tracers need to be transported. On-demand exchange is a way to reduce the communication of halo exchange for the SL method and will be investigated in a future study.

Significant differences in the communication times of the wide halo exchange of 20 grid points for topology torus-L, fattree-L, and dragonfly-ML are shown in Fig. 9b. It can be seen that topology torus- $\mathrm{L}$ performs the worst, fattree- $\mathrm{L}$ is the best, and the performance of dragonfly-ML is between that of torus- $\mathrm{L}$ and fattree-L. The communication time of the wide halo exchange of 20 grid points for the topology tour-L abruptly increases at approximately $10^{3}$ MPI processes and then gradually decreases when the number of MPI tasks becomes larger than $3 \times 10^{3}$ MPI processes. The impact of the routing algorithm on the communication time of the wide halo exchange of 20 grid points (Fig. 9c) is the same as on that of transposition (Fig. 5d): the routing algorithm valiant performs the best, the routing algorithm ugal performs the worst, and the routing algorithm minimal is between valiant and ugal.

\subsection{Allreduce in Krylov subspace methods for the semi-implicit method}

If, on average, the GCR with a restart number $k=3$ is convergent with $N=25$ iterations, the number of allreduce calls is $2 \times N=50$. The black and blue lines are the communication times of 50 allreduce operations using MPI_Allreduce and the recursive- $k$ algorithm, respectively, that is, the estimated communication time of one single GCR call (Fig. 10a). Contrary to that of transposition, the com- 

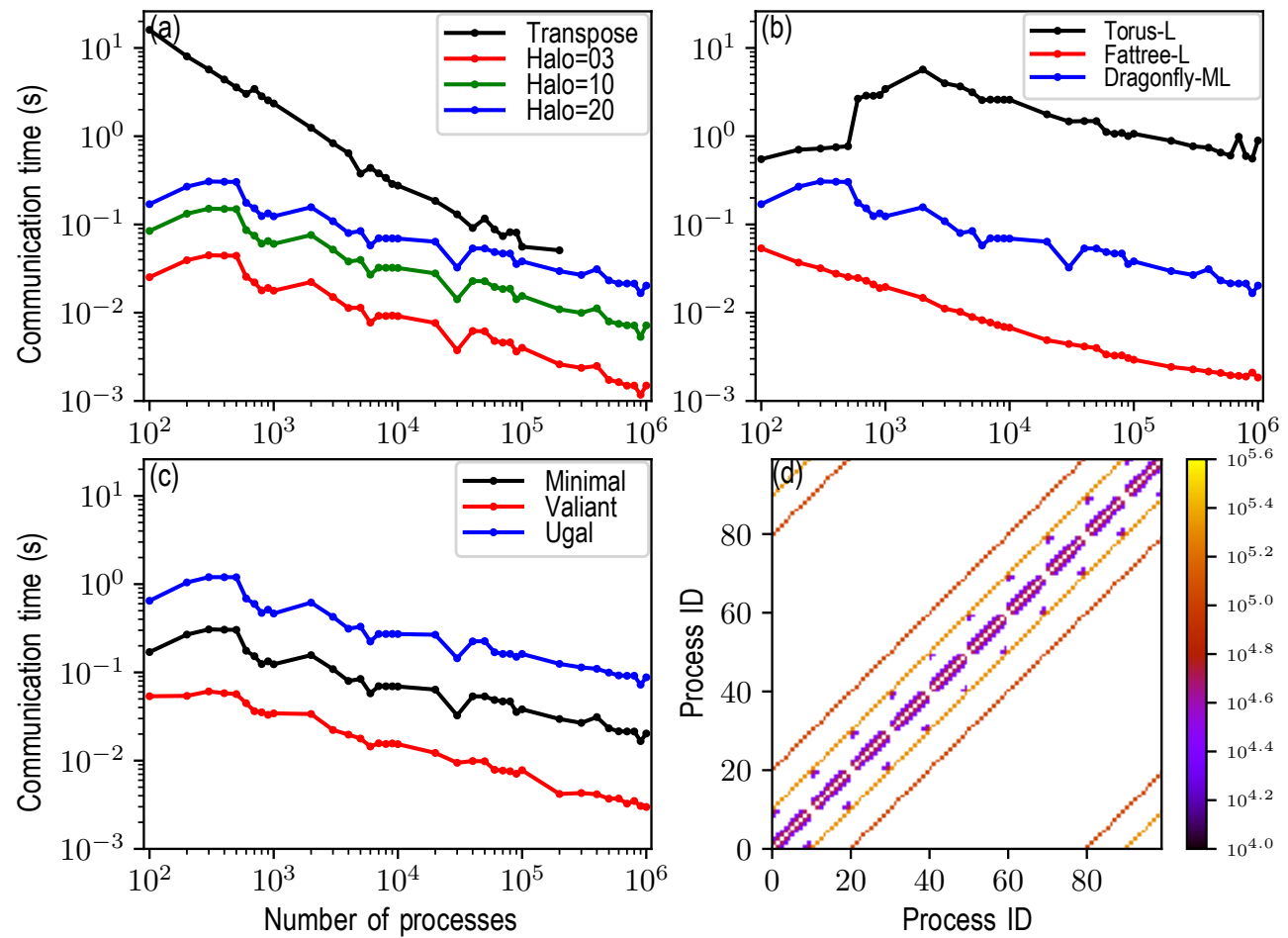

Figure 9. Panel (a) is the communication times of the halo exchange with a halo of 3 (red line), 10 (green line), and 20 (blue line) grid points, and the communication time of transposition for the spectral transform method is shown for comparison (black line). Panel (b) is the communication times of the halo exchange with a halo of 20 grid points for the topology of torus-L (black line), fattree-L (red line), and dragonfly-ML (blue line). Panel (c) is the communication times of the halo exchange with a halo of 20 grid points for the routing algorithm of minimal (black line), valiant (red line), and ugal (blue line). Panel (d) illustrates the communication pattern of the halo exchange with a wide halo. The circle markers in (a)-(c) indicate the numbers of processes of the corresponding simulations.

munication time of GCR increases as the number of MPI processes increases. Following the trend, the communication of a single GCR call may be similar to or even larger than that of a single transposition when the number of MPI processes approaches to or is beyond 1 million. Although it is believed that the spectral method does not scale well owing to its time-consuming transposition, it does not suffer from this expensive allreduce operation for the SI method because of its mathematical advantage that spherical harmonics are the eigenfunctions of Helmholtz operators. In this sense, a grid-point model with the SI method in which the threedimensional Helmholtz equation is solved by Krylov subspace methods may also not scale well at the exascale unless the overhead of allreduce communication can be mitigated by overlapping it with computation (Sanan et al., 2016).

Figure $10 \mathrm{~b}$ shows the communication times of allreduce operations using the recursive- $k$ algorithm on the topologies of torus-L, fattree-L, and dragonfly-ML. The impact of topology on the communication performance of allreduce operations is obvious. The topology of torus-L has the best performance but is similar to that of dragonfly-ML for more than $5 \times 10^{5}$ MPI processes; fattree-L has the worst performance. However, the three routing algorithms (minima, valiant, and ugal) for the dragonfly-ML topology have a negligible impact on the communication performance of allreduce operations (figure not shown); this may be because of the tiny messages (only three doubles for the restart number $k=3$ ) communicated by the allreduce operation.

One advantage of the recursive- $k$ algorithm of the allreduce operation is that the radix $k$ can be selected to reduce the stages of communication by making full use of the bandwidth of the underlying interconnect network. We repeat the experiment, whose configuration is as that of the blue line in Fig. 10a, for the proper radix $k \in[2,32]$, and the optimal radix is that with the lowest communication time for a given number of MPI processes. For each number of MPI processes, there is an optimal radix. The statistics of all the optimal radices are shown in Fig. 10c. It can be seen that the minimum and maximum optimal radices are 5 and 32 , respectively. Thus, the recursive doubling algorithm that is equivalent to the recursive- $k$ algorithm with radix $k=2$ is not efficient since the optimal radix is at least 5 . The median number of optimal radices is approximately 21 , and the mean number is less than but very close to the median number. We cannot derive an analytic formula for the optimal radix since modelling the congestion is difficult in an analytic 
model. However, for a given resolution of NWP model and a given HPC system, fortunately, the number of processes, bandwidth, and latency are fixed; thus, it is easy to perform experiments to obtain the optimal radix.

\section{Conclusion and discussion}

This work shows that it is possible to make simulations of the MPI patterns commonly used in NWP models using very large numbers of MPI tasks. This opens up the possibility of examining and comparing the impact of different factors such as latency, bandwidth, routing, and network topology on response time. We have provided an assessment of the performance and scalability of three key MPI operations in an atmospheric model at the exascale by simulating their skeleton programs on an SST/macro simulator. After optimization of the memory and efficiency of the SST/macro simulator and construction of the skeleton programs, a series of experiments was carried out to investigate the impacts of the collective algorithm, the topology and its configuration, the routing algorithm, the bandwidth, and the latency on the performance and scalability of transposition, halo exchange, and allreduce operations. The experimental results show the following:

1. The collective algorithm is extremely important for the performance and scalability of key MPI operations in the atmospheric model at the exascale because a good algorithm can make full use of the bandwidth and reduce the stages of communication. The generalized ring- $k$ algorithm for the alltoallv operation and the generalized recursive- $k$ algorithm for the allreduce operation proposed herein perform the best.

2. Topology, its configuration, and the routing algorithm have a considerable impact on the performance and scalability of communications. The fattree topology usually performs the best, but its scalability becomes weak with a large number of MPI processes. The dragonfly topology balances the performance and scalability well, and can maintain almost the same scalability with a large number of MPI processes. The configurations of the dragonfly topology indicate that a proper configuration can be used to avoid the hotspots of congestion and lead to good performance. The minimal routing algorithm is intuitive and performs well. However, the valiant routing algorithm (which randomly chooses an intermediate node to uniformly disperse the communication over the network to avoid the hotspots of congestion) performs much better for heavy congestion.

3. Although they have an important impact on communication, bandwidth and latency cannot be infinitely grown and reduced owing to the limitation of hardware. Thus, it is important to design innovative algorithms to make full use of the bandwidth and to reduce the effect of latency.

4. It is generally believed that the transposition for the spectral transform method, which is a multiple simultaneous all-to-all personalized communication, poses a great challenge to the scalability of the spectral model. This work shows that the scalability of the spectral model is still acceptable in terms of MPI transposition. However, the wide halo exchange for the semiLagrangian method and the allreduce operation in the GCR iterative solver for the semi-implicit method, both of which are often adopted by the grid-point model, also face the stringent challenge of scalability at the exascale.

In summary, both software (algorithms) and hardware (characteristics and configuration) are of great importance to the performance and scalability of the atmospheric model at the exascale. The software and hardware must be codesigned to address the challenge of the atmospheric model for exascale computing.

As shown previously, the communications of the wide halo exchange for the semi-Lagrangian method and the allreduce operation in the GCR iterative solver for the semi-implicit method are expensive at the exascale. The on-demand halo exchange for the semi-Lagrangian and the pipeline technique to overlap with the communication with the computation for the GCR iterative solver are not researched in this study and should be investigated. All the compute nodes in this work only contain one single-core CPU, which is good for assessing the communication of the interconnect network; however, the architectures of current and future supercomputers are multi-core and multi-socket nodes, even in nonCPU architectures. These more complex hierarchies seem to complicate the inter-process communications. However, an MPI rank can be bound to any core for multi-core and multisocket nodes. For example, an MPI rank can be bound to any processor/co-processor for MIC (many-integrated core) architectures such as Xeon Phi using the INTEL MPI library, and an MPI rank can be bound to a CPU core but can communicate with GPUs (graphics processing units) for GPU architectures using a CUDA-aware (compute unified device architecture) MPI. Because a multi-core node behaves more or less like a more powerful single-core node when OpenMP (open multi-processing) is used for the intra-node parallelization, the conclusions in this study could be generalized to the complex hierarchical system. Multiple MPI processes per node may be good for the local pattern communication such as thin halo exchange since the shared memory communication mechanism is used but may result in congestion in the network interface controller for inter-node communication. The congestion can be mitigated or even eliminated if each node has more network interface controllers (NICs) or a network interface controller with multi-ports (as a mini-switch). From this point of view, the conclusions should still be valid 

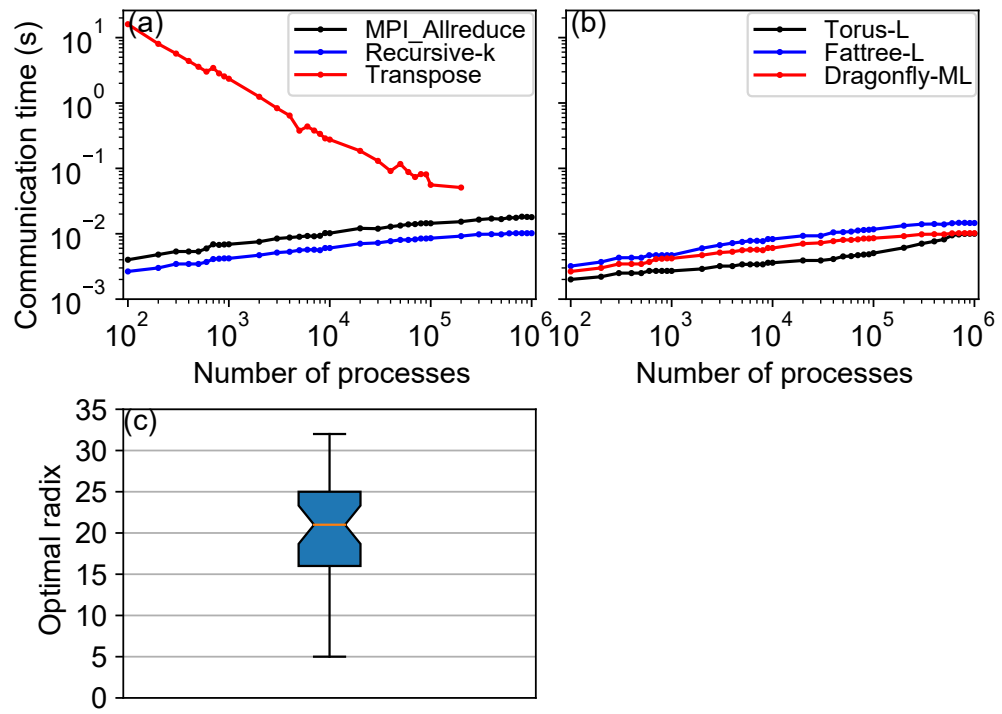

Figure 10. Panel (a) is the communication times of the allreduce operation using the MPI_Allreduce (black line) and the recursive- $k$ algorithm (blue line), and the communication time of transposition for the spectral transform method is shown for comparison (red line). Panel (b) is the communication times of the allreduce operation using the recursive- $k$ algorithm for the topology torus- $\mathrm{L}$ (black line), fattree- $\mathrm{L}$ (blue line), and dragonfly-ML (red line). Panel (c) is the statistics of the optimal radices for the recursive- $k$ algorithm. The circle markers in (a)-(b) indicate the numbers of processes of the corresponding simulations.

for the complex hierarchical architectures, but the scalability might be affected. The more MPI processes, the less computation per node if there is only one single-core CPU per node; thus, computation is not considered in this paper. Because multi-core or many-core processors share a memory bus, it is possible for a memory-intensive application (such as an atmospheric model) to saturate the memory bus and result in degraded performances of all the computations running on that processor. The assessment of computations is currently underway and a detailed paper will be presented separately; the purpose of this subsequent study is to model the time response of a time step of a model such as the regional model (AROME) used by Météo France.

Code availability. The code of the SST/macro simulator is publicly available at https://github.com/sstsimulator/sst-macro (Sandia National Laboratories, 2018). The skeleton programs, scripts, and our modified version of SST/macro 7.1.0 for the simulations presented in this paper are available at https://doi.org/10.5281/zenodo.1066934 (Zheng, 2017).

Competing interests. The authors declare that they have no conflict of interest.

Acknowledgements. This work was supported by the ESCAPE (Energy-efficient Scalable Algorithms for Weather Prediction at Exascale) project. The ESCAPE project has received funding from the European Union's Horizon 2020 research and innovation programme under grant agreement no. 671627. Our sincere gratitude goes to two anonymous reviewers and the topical editor David Ham for their thoughtful comments and suggestions that have helped improve this paper substantially.

Edited by: David Ham

Reviewed by: two anonymous referees

\section{References}

Acun, B., Jain, N., Bhatele, A., Mubarak, M., Carothers, C. D., and Kale, L. V.: Preliminary Evaluation of a Parallel Trace Replay Tool for HPC Network Simulations, Springer International Publishing, Cham, 417-429, 2015.

Ajima, Y., Sumimoto, S., and Shimizu, T.: Tofu: A 6D Mesh/Torus Interconnect for Exascale Computers, Computer, 42, 36-40, 2009.

Alverson, B., Froese, E., Kaplan, L., and Roweth, D.: Cray XC Series Network, Cray Inc., 2015.

Barros, S. R. M., Dent, D., Isaksen, L., Robinson, G., Mozdzynski, G., and Wollenweber, F.: The IFS model: a parallel production weather code, Parallel Comput., 21, 1621-1638, 1995.

Bauer, P., Thorpe, A., and Brunet, G.: The quiet revolution of numerical weather prediction, Nature, 525, 47-55, 2015.

Böhm, S. and Engelmann, C.: xSim: The extreme-scale simulator, in: 2011 International Conference on High Performance Computing Simulation, 280-286, 2011.

Casanova, H., Gupta, A., and Suter, F.: Toward More Scalable OffLine Simulations of MPI Applications, Parallel Processing Letters, 25, 1541002, https://doi.org/10.1142/S0129626415410029, 2015 . 
Dechev, D. and Ahn, T. H.: Using SST/Macro for Effective Analysis of MPI-Based Applications: Evaluating Large-Scale Genomic Sequence Search, IEEE Access, 1, 428-435, 2013.

Degomme, A., Legrand, A., Markomanolis, G. S., Quinson, M., Stillwell, M., and Suter, F.: Simulating MPI Applications: The SMPI Approach, IEEE T. Parall. Distr., 28, 2387-2400, 2017.

Dubos, T., Dubey, S., Tort, M., Mittal, R., Meurdesoif, Y., and Hourdin, F.: DYNAMICO-1.0, an icosahedral hydrostatic dynamical core designed for consistency and versatility, Geosci. Model Dev., 8, 3131-3150, https://doi.org/10.5194/gmd-8-3131-2015, 2015.

Ehrendorfer, M.: Spectral numerical weather prediction models, SIAM, 482 pp., https://doi.org/10.1137/1.9781611971996, 2012.

Eisenstat, S. C., Elman, H. C., and Schultz, M. H.: Variational iterative methods for nonsymmetric systems of linear equations, SIAM J. Numer. Anal., 20, 345-357, 1983.

Engelmann, C.: Scaling to a million cores and beyond: using lightweight simulation to understand the challenges ahead on the road to exascale, Future Gener. Comp. Sy., 30, 59-65, 2014.

Hoefler, T., Schneider, T., and Lumsdaine, A.: LogGOPSim - Simulating Large-Scale Applications in the LogGOPS Model, in: Proceedings of the 19th ACM International Symposium on High Performance Distributed Computing, 597-604, ACM, 2010.

Hortal, M.: The development and testing of a new two-time-level semi-Lagrangian scheme (SETTLS) in the ECMWF forecast model, Q. J. Roy. Meteor. Soc., 128, 1671-1687, 2002.

Hoskins, B. J. and Simmons, A. J.: A multi-layer spectral model and the semi-implicit method, Q. J. Roy. Meteor. Soc., 101, 637-655, 1975.

Jain, N., Bhatele, A., White, S., Gamblin, T., and Kale, L. V.: Evaluating HPC Networks via Simulation of Parallel Workloads, in: SC16: International Conference for High Performance Computing, Networking, Storage and Analysis, 154-165, 2016.

Janssen, C. L., Adalsteinsson, H., Cranford, S., Kenny, J. P., Pinar, A., Evensky, D. A., and Mayo, J.: A Simulator for Large-Scale Parallel Computer Architectures, International Journal of Distributed Systems and Technologies, 1, 57-73, 2010.

Juang, H. H., Hong, S., and Kanamitsu, M.: The NCEP regional spectral model: an update, B. Am. Meteorol. Soc., 78, 21252143, 1997.

Kanamitsu, M., Kanamaru, H., Cui, Y., and Juang, H.: Parallel implementation of the regional spectral atmospheric model, Tech. rep., Scripps Institution of Oceanography, University of California at San Diego, and National Oceanic and Atmospheric Administration for the California Energy Commission, PIER EnergyRelated Environmental Research, cEC-500-2005-014, 2005.

Kim, J., Dally, W. J., Scott, S., and Abts, D.: Technology-Driven, Highly-Scalable Dragonfly Topology, in: 2008 International Symposium on Computer Architecture, 77-88, 2008.

Lagadapati, M., Mueller, F., and Engelmann, C.: Benchmark Generation and Simulation at Extreme Scale, in: 2016 IEEE/ACM 20th International Symposium on Distributed Simulation and Real Time Applications (DS-RT), 9-18, 2016.

Leiserson, C. E.: Fat-trees: Universal networks for hardwareefficient supercomputing, IEEE T. Comput., C-34, 892-901, 1985.

Li, L., Xue, W., Ranjan, R., and Jin, Z.: A scalable Helmholtz solver in GRAPES over large-scale multicore cluster, Concurr. Comp.Pract. E., 25, 1722-1737, 2013.
Lin, S.-J.: A “vertically Lagrangian" finite-volume dynamical core for global models, Mon. Weather Rev., 132, 2293-2307, 2004.

Mubarak, M., Carothers, C. D., Ross, R. B., and Carns, P.: Enabling Parallel Simulation of Large-Scale HPC Network Systems, IEEE T. Parallel Distr., 28, 87-100, 2017.

Noeth, M., Ratn, P., Mueller, F., Schulz, M., and de Supinski, B. R.: ScalaTrace: Scalable compression and replay of communication traces for high-performance computing, J. Parallel Distr. Com., 69, 696-710, 2009.

Núñez, A., Fernández, J., Garcia, J. D., Garcia, F., and Carretero, J.: New techniques for simulating high performance MPI applications on large storage networks, J. Supercomput., 51, 40-57, 2010.

Qaddouri, A. and Lee, V.: The Canadian global environmental multiscale model on the Yin-Yang grid system, Q. J. Roy. Meteor. Soc., 137, 1913-1926, 2011.

Ritchie, H.: Implementation of the Semi-Lagrangian Method in a High-Resolution Version of the ECMWF forecast model, Mon. Weather Rev., 123, 489-514, 1995.

Robert, A., Henderson, J., and Turnbull, C.: An implicit time integration scheme for baroclinic models of the atmosphere, Mon. Weather Rev., 100, 329-335, 1972.

Sanan, P., Schnepp, S. M., and May, D. A.: Pipelined, flexible krylov subspace methods, SIAM J. Sci. Comput., 38, C441C470, 2016.

Sandbach, S., Thuburn, J., Vassilev, D., and Duda, M. G.: A SemiImplicit version fo the MPAS-atmosphere dynamical core, Mon. Weather Rev., 143, 3838-3855, 2015.

Satoh, M., Matsuno, T., Tomita, H., Miura, H., Nasuno, T., and Iga, S.: Nonhydrostatic icosahedral atmospheric model (NICAM) for global cloud resolving simulations, J. Comput. Phys., 227, 34863514, 2008.

Seity, Y., Brousseau, P., Malardel, S., Hello, G., Bénard, P., Bouttier, F., Lac, C., and Masson, V.: The AROME-France ConvectiveScale Operational Model, Mon. Weather Rev., 139, 976-991, 2011.

Skamarock, W. C., Klemp, J. B., Duda, M. G., Fowler, L. D., and Park, S.-H.: A multiscale nonhydrostatic atmospheric model using centroidal voronoi tesselations and C-grid staggering, Mon. Weather Rev., 140, 3090-3105, 2012.

Smolarkiewicz, P. K., Deconinck, W., Hamrud, M., Kühnlein, C., Mozdzynski, G., Szmelter, J., and Wedi, N. P.: A finite-volume module for simulating global all-scale atmospheric flows, J. Comput. Phys., 314, 287-304, https://doi.org/10.1016/j.jcp.2016.03.015, 2016.

SNL, L. C.: SST/macro 7.1: User's Manual, Sandia National Labs, Livermore, CA, 2017.

Staniforth, A. and Côté, J.: Semi-Lagrangian integration schemes for atmospheric models-a review, Mon. Weather Rev., 119, 2206-2223, 1991.

Temperton, C.: Self-sorting mixed-radix fast Fourier transforms, J. Comput. Phys., 52, 1-23, 1983.

Thakur, R., Rabenseifner, R., and Gropp, W.: Optimization of Collective Communication Operations in MPICH, Int. J. High Perform. Comput. Appl., 19, 49-66, 2005.

Tikir, M. M., Laurenzano, M. A., Carrington, L., and Snavely, A.: PSINS: An Open Source Event Tracer and Execution Simulator for MPI Applications, pp. 135-148, Springer Berlin Heidelberg, Berlin, Heidelberg, 2009. 
Wedi, N. P.: Increasing horizontal resolution in numerical weather prediction and climate simulations: illusion or panacea?, Philos. T. R. Soc. A, 372, 20130289, https://doi.org/10.1098/rsta.2013.0289, 2014.

Wedi, N. P., Hamrud, M., and Mozdzynski, G.: A fast spherical harmonics transform for global NWP and climate models, Mon. Weather Rev., 141, 3450-3461, 2013.

Wike, J. J. and Kenny, J. P.: Using Discrete Event Simulation for Programming Model Exploration at Extreme-Scale: Macroscale Components for the Structural Simulation Toolkit (SST), Tech. rep., Sandia National Laboratories, sAND2015-1027, 2014.

Wolfe, N., Carothers, C. D., Mubarak, M., Ross, R., and Carns, P.: Modeling a Million-Node Slim Fly Network Using Parallel Discrete-Event Simulation, in: Proceedings of the 2016 Annual ACM Conference on SIGSIM Principles of Advanced Discrete Simulation, 189-199, ACM, 2016.

Zahavi, E., Johnson, G., Kerbyson, D. J., and Lang, M.: Optimized InfiniBand $^{\mathrm{TM}}$ fat-tree routing for shift all-to-all communication patterns, Concurr. Comp.-Pract. E., 22, 217-231, 2010.
Zangl, G., Reinert, D., Ripodas, P., and Baldauf, M.: The ICON (icosahedral non-hydrostatic) modelling framework of DWD and MPI-M: description of the non-hydrostatic dynamical core, Q. J. Roy. Meteor. Soc., 141, 563-579, 2015.

Zerroukat, M. and Allen, T.: A three-dimensional monotone and conservative semi-Lagrangian scheme (SLICE-3D) for transport problems, Q. J. Roy. Meteor. Soc., 138, 1640-1651, 2012.

Zheng, Y.: Performance and Scalability of Communications in Atmospheric Model for Exascale Supercomputer, Zenodo, https://doi.org/10.5281/zenodo.1066934, 2017.

Zheng, G., Kakulapati, G., and Kale, L. V.: BigSim: a parallel simulator for performance prediction of extremely large parallel machines, in: 18th International Parallel and Distributed Processing Symposium, 2004. Proceedings, 78 pp., 2004. 